\title{
Design and fabrication of inclined arm miniature sized quadcopter UAV
}

\author{
Vimal Raj $\mathrm{V}^{1}$, Sriram $\mathrm{S}^{2}$, Ram Mohan $\mathrm{P}^{3}$, Manoj Austin $\mathrm{T}^{4}$

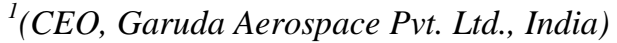 \\ ${ }_{2}^{2}$ (Design Engineer, Garuda Aerospace Pvt. Ltd., India) \\ ${ }^{3}$ (Design Engineer, Garuda Aerospace Pvt. Ltd., India) \\ ${ }^{4}$ (Design Engineer, Garuda Aerospace Pvt. Ltd., India)
}

\begin{abstract}
Quadcopter UAV also known as quadrotor is the next form of helicopters having more dynamic stability than helicopters. They play a predominant role in different areas like surveillance, military operations, fire sensing and some important areas having many complexities. Quadcopters are unmanned aerial vehicles with ability of vertical takeoffs, landings and hovering at a desired location. This research paper addresses the design and development of an inclined arm quadcopter for mini payload and longtime endurance.
\end{abstract}

Keywords: Inclined Arm, UAV and Quadcopter.

\section{Introduction}

Quadcopters also known as quad rotors or multirotor aircrafts are emerging favorites in unmanned aerial vehicle (UAV) design. This is due to their ability to hover in congested areas along with vertical takeoff and landing (VTOL) capability. Quadcopter consist of four rotors which are fixed at the end of the frame structure. In this case, a pair of rotors in one arm facing each other rotates in clockwise direction while the adjacent pair of rotors rotates in anticlockwise direction. Hence the resultant torque acting on the air frame structure is zero.

\subsection{Inclined Arm UAV}

The inclined arm UAV is one of the modification given to the airframe structure. In this Quadcopter the arms are inclined with respect to motors. For this design we are evaluating the thrust, maneuverability, forces acting etc.

\subsection{Design requirements:}

\section{Design Development}

In order to design the Quad copter, for miniature payload applications, following requirements has to be achieved.

- The Centre plate should be less than $300 \mathrm{~mm}$ x $300 \mathrm{~mm}$.

- The Quadcopter should carry a maximum payload of $1 \mathrm{~kg}$.

- Necessary power system has to be selected.

- The total weight of the quad should be less than $3 \mathrm{~kg}$.

- And the design should be done in such a way that the arm should be inclined to a particular angle.

\subsection{Centre frame}

The Centre frame is the base of the quadcopter which carries the whole weight of the copter. The design should be rigid and strong enough to carry all the weight including motor, propeller, payload and all other structural members. The Centre plate is designed in such a way that the arms are inclined to the center frame which is shown below in Fig 2.1. It is designed in such a way that the arms are inclined to the Centre frame.

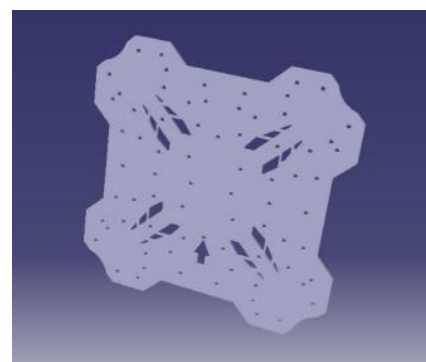

Fig 2.1: Centre Plate 


\subsection{Copter Arm:}

The arm should be in length to have an enough space between propellers. The arm should not exceed the maximum length so that the load acting on the Centre plate should not exceed the maximum load failure. The copter arm design is shown in Fig 2.2.

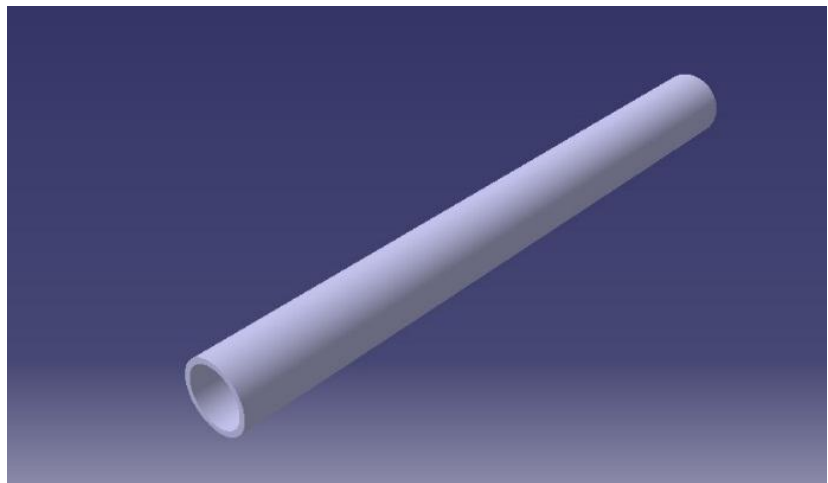

Fig 2.2: Quadcopter Arm

\subsection{Propeller}

The propeller is one of the important part of the quad copter thrust generating unit. The propeller is designed based on the airfoil cross section, pitch angle, root to tip diameter and the twist of the propeller. The diameter of the propeller and the rpm of the motor is the most important thrust factors. Propeller design is shown below in Fig 2.3.

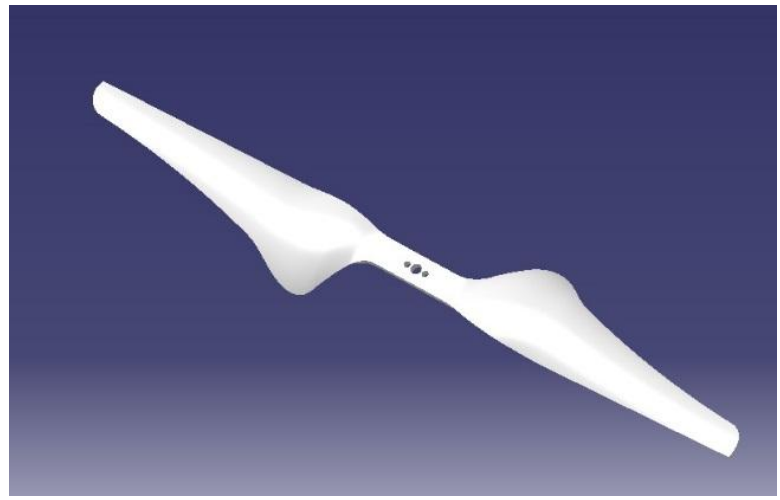

Fig 2.3: Propeller

\subsection{Landing Clamp and Arm:}

Landing gear is the bottom part of the copter which will contact with the landing and carries whole weight. The landing gear is in particular angle so that the quadcopter will land safely in properly balanced manner and should be long and strong enough to carry the whole weight of the copter. The landing clamp and arm is shown in Fig 2.4.

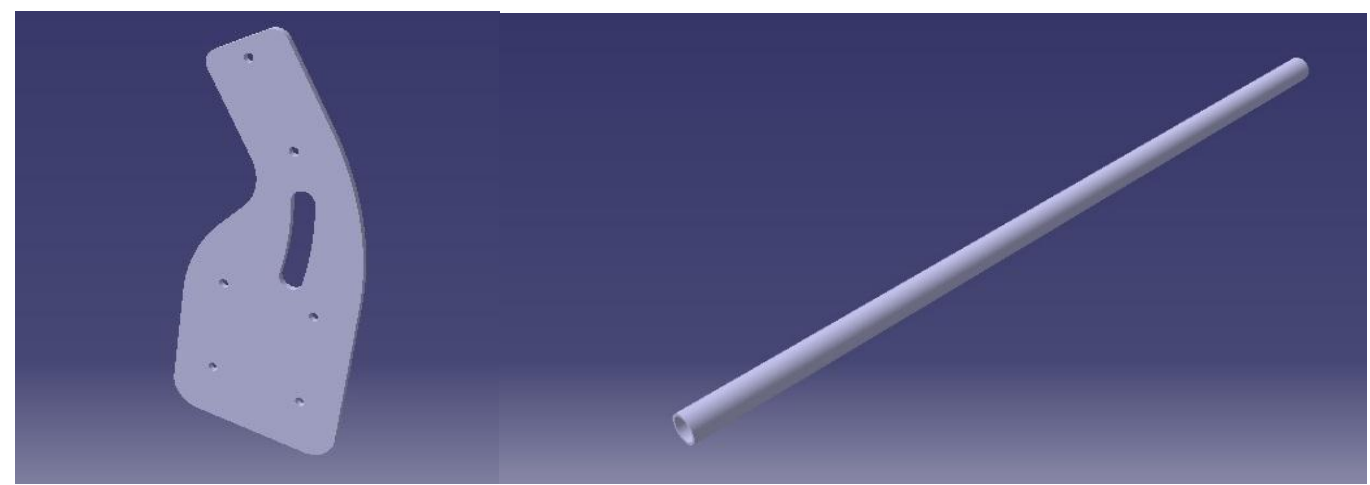

fig 2.4: Landing Arm and Clamp 


\subsection{Final Assembly}

All individual parts are assembled together, the final assembly of the copter is done based on the requirement and innovation included by the designer to implement the idea. Assembled Final design of the inclined arm Quadcopter is shown below in Fig 2.5.

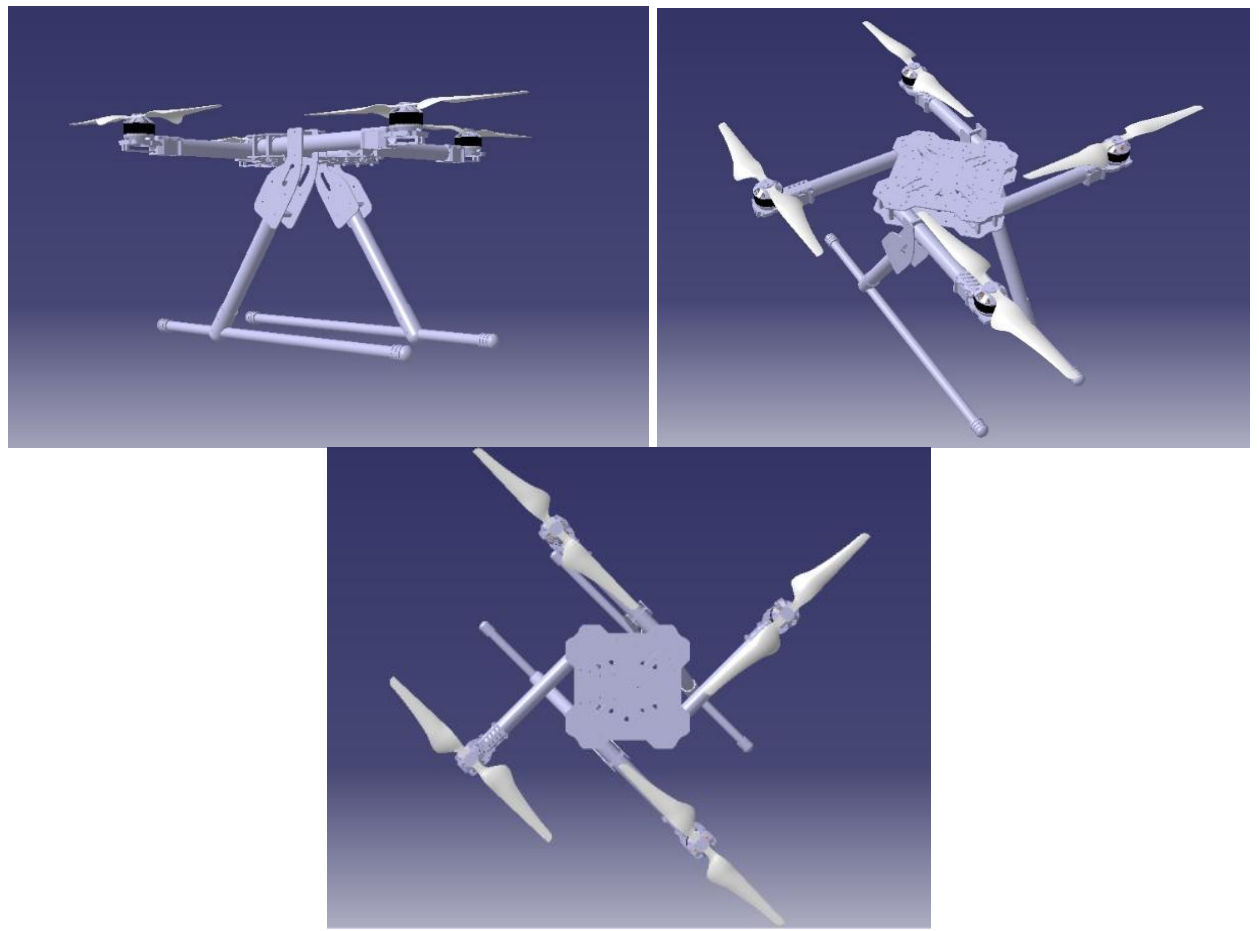

Fig 2.5: Final assembly Inclined Arm Quadcopter

\section{Fabrication}

A composite is a mixture of material made from two or more constituent materials with significantly different physical or chemical properties that, when combined, produce a new material with characteristics different from the individual components.

Newer constituents to mixture is selected based on many reasons, which are

- stronger,

- lighter,

- Less expensive when compared to traditional materials.

\subsection{Material selection:}

The frame should have low weight and sufficient strength to bear the static and dynamic loading condition without any kind of fracture. The quadcopter which is fully made of composites which greatly reduces the weight of the copter which increases the thrust to weight ratio and also increases the endurance of the copter.

Based on the requirements, the materials is selected, most commonly composites are used because of their advantages over metals. The designed Quadcopter is fabricated, all avionics components are integrated onboard and is prepared for testing. The final product is shown in Fig 3.1.

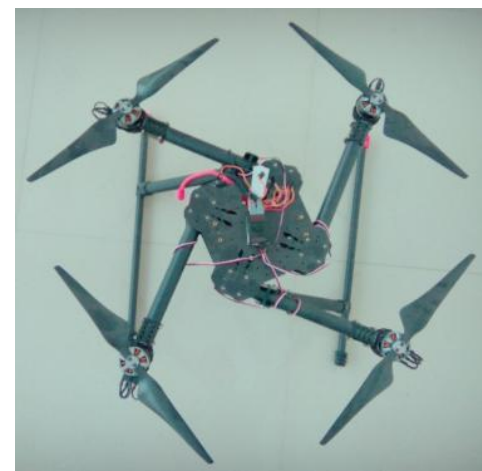

Fig 3.1 (a) Top View

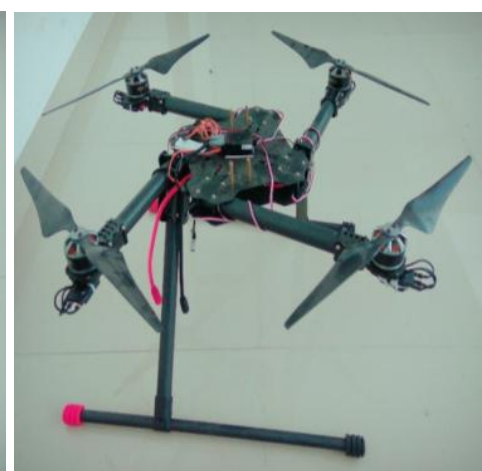

(b) Isometric View 


\section{Testing And Observation}

The newly fabricated quadcopter was assembled with all required avionics equipments. Testing of Quad was done by flying it in safe and controlled outdoor environment. The performance of inclined arm quadcopter is similar to ordinary quadcopter. The maneuverability and stability of the new quad is slightly deviated from the conventional design. Further innovation is required to analyze the performance.

\section{Journal Papers:}

\section{References}

[1]. P. Pounds and R. Mahony, "Design principles of large quadrotors for practical applications" in Proceedings of the IEEE International Conference on Robotics and Automation (ICRA), pp. 3265 -3270, May 2009

[2]. Corey Russel et al, Journal of Design and Realization of intelligent portable Aerial surveillance system 43, pp.1923-1930 (2010)

[3]. Felipe Bohorquez, Design, Analysis and Hover performance of a Rotary Wing Micro Air Vehicle, 2000.

[4]. P. Pounds, R. Mahony, and P. Corke, "Modelling and Control of a Large Quadrotor Robot," in Control Engineering Practice, vol. 18, pp. $691-699,2010$.

\section{Books:}

[5]. John David Anderson, Fundamentals of Aerodynamics (McGraw-Hill Publication).

\section{Proceedings papers:}

[6]. Samir Bouabdallah, Design and Control of an Indoor Micro Quadrotor, recent progress in sensor technology, 2002. 